\title{
Frontières
}

\section{Patrick Baudry, La place des morts, Paris, Armand Colin, 1999, 201 pages}

\section{Sébastien St-Onge}

Volume 12, numéro 2, printemps 2000

URI : https://id.erudit.org/iderudit/1074411ar

DOI : https://doi.org/10.7202/1074411ar

Aller au sommaire du numéro

Éditeur(s)

Université du Québec à Montréal

ISSN

1180-3479 (imprimé)

1916-0976 (numérique)

Découvrir la revue

Citer ce compte rendu

St-Onge, S. (2000). Compte rendu de [Patrick Baudry, La place des morts, Paris, Armand Colin, 1999, 201 pages]. Frontières, 12(2), 89-90.

https://doi.org/10.7202/1074411ar

Ce document est protégé par la loi sur le droit d'auteur. L'utilisation des services d'Érudit (y compris la reproduction) est assujettie à sa politique d'utilisation que vous pouvez consulter en ligne.

https://apropos.erudit.org/fr/usagers/politique-dutilisation/
Cet article est diffusé et préservé par Érudit.

Érudit est un consortium interuniversitaire sans but lucratif composé de l’Université de Montréal, l’Université Laval et l'Université du Québec à Montréal. Il a pour mission la promotion et la valorisation de la recherche. https://www.erudit.org/fr/ 
Ne retenons que le positif, comme si les liens avec le passé (ou le trépassé) ouvraient l'avenir." (p. 66). $\dot{A}$ ce titre, des secondes obsèques sont proposées. Or la tristesse n'est pas autorisée, puisque le mort dispose d'un ministre pour compter auprès de ses survivants: "au notaire est dévolue la tâche la plus importante (par quelque côtés résurrectionniste); il s'inquiète de la matérialité des biens (là où le défunt s'est inscrit et a en quelque sorte déposé son ingéniosité, parce que la terre du paysan [on souhaite qu'il y ait là une allégorie], avant d'être une surface, expose la manière dont il l'a travaillée et sans doute améliorée), ainsi que du témoignage écrit de ses volontés. II permet au mort non seulement de revenir dans la société mais de la consolider ou de la remodeler (il suscite de nouvelles ententes). La relation des vivants et des morts est rétablie, matériellement et spirituellement assurée.» (p. 75). Cette "indéchirable liaison» entre les vivants et les morts est toutefois malmenée: sont fustigés l'individualisme, le libéralisme, la gestion du temps qui rejette le mourant, etc. et "l'exaltation des différences (qui conduisent elles-mêmes aux différents.)" (p. 79). Que fait d'autre l'auteur? Concluons rapidement: il est désolant que cette perspective communautaire, si pertinente et potentiellement multivoque, soit si peu argumentée.

Il est souligné en couverture du livre qu'il semblait aux auteurs "que les textes actuellement disponibles étaient insuffisants: le deuil était envoyé à une question psychologique, à la souffrance psychique.". S'il est exact qu'on tend à cantonner le deuil dans une "ingénierie" psychosociale, ou une pathologisation (et ce ne sont pas tous les écrits et pratiques en psychanalyse et en psychiatrie qui distillent ce travers!), on reste encore ici néanmoins perplexe: quelle littérature a été consultée, à travers autant des ouvrages collectifs récents que des ouvrages majeurs d'origine francophone et anglosaxonne? Comment autant parler de mémoire - de vie des morts - et à ce point négliger ses ascendants? Il ne s'agit pas tant d'effectuer un repérage académique des idées, mais au moins d'évoquer d'autres penseurs contemporains sur ces questions précises, desquels on pourrait bien être cryptomnésiques. Le graphisme et la couleur carillonante de couverture de ce petit ouvrage semblent nous lancer: morts, les Foucault, Jankélévitch, Thomas (pour ne citer que des philosophes de formation: et puis Baudrillard, et encore, que du côté français!) et leurs gros pavés ardus et inspirants... En ce sens, la prétention du titre de l'ouvrage et son propos, ce dernier certes condensé et stimulant dans le sens de provocateur, porte plus au scepticisme sympathique qu'à la validation scientifique: car à côté de ce que l'on peut en dégager ici, en partie fondé, cet "autrement " peut être différent que celui appelé, moins monolithique et tout de même porté par le savoir dit populaire, dans les récits de bord de route, dans les évocations en thérapie (hé oui!), dans les pratiques sociales nouvelles encore peu sondées, dans les chansons... Reprendre tout à coup cet "autrement" à son propre compte serait éloquent de quels enjeux actuels autour des discours - devenus "productions» sur la mort?

\section{Luce Des Aulniers}

\section{Patrick Baudry \\ La place des morts}

Paris, Armand Colin, 1999,

201 pages.

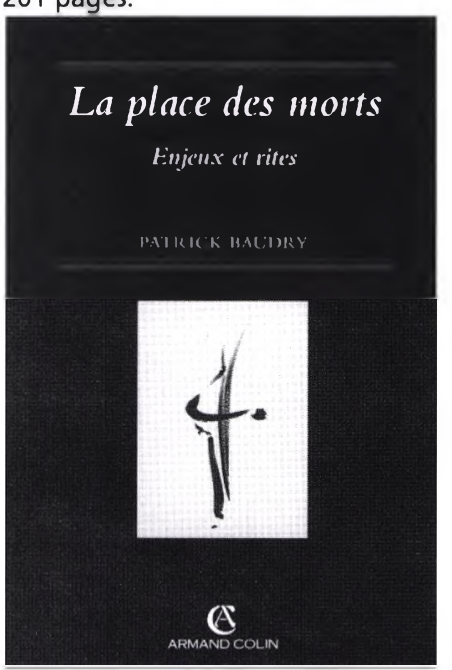

À une époque où il faut tout voir pour croire, intellectualiser avant d'acquiescer, comment peut se construire l'espace des morts, cet espace qui sollicite obligatoirement un imaginaire collectif? La modernité a-t-elle réussi à ravaler la dimension fondamentale de la mort à un événement où l'individu pourrait, à lui seul, négocier son rapport à l'altérité? Nos rituels funéraires sont-ils devenus le lieu d'une parole désincarnée, relégués à l'expression de politesses circonstancielles? Voilà autant de questions essentielles auxquelles nous convie l'ouvrage du sociologue Patrick Baudry.

C'est à travers l'étude d'une culture quotidienne, où les gestes discrets et les paroles des survivants témoignent de la négociation perpétuelle s'établissant entre vivants et morts, que le sociologue entend démontrer comment la culture contemporaine résiste à la production d'une société sans altérité. Une société qui voudrait communiquer sur l'incommunicable, réduire la place des morts aux cimetières et colmater les vides béants laissés par la mort. Mais, nous dit l'auteur, en creux de cette rationalisation de la mort et loin des lieux communs, un récit discret prend vie entre vivants et morts.

Avant de conduire le lecteur sur ce versant peu exploré, de prendre acte d'une parole souvent en morceaux et de tendre l'oreille aux murmures du silence, l'auteur nous rappelle les enjeux des rites funéraires. Le détour théorique qu'il emprunte permet au lecteur de saisir l'évolution et les mutations de la ritualite funéraire contemporaine. La démonstration exhaustive, qui s'appuie essentiellement sur l'enseignement de Louis-Vincent Thomas, amène le sociologue à identifier les misères de la ritualité funéraire qui ferait état d'une société à la fois en panne de sens et de solidarité. L'inquiétude de l'auteur se traduit en ces termes:" N'est-ce pas à une ritualité de la ritualité qu'on a de plus en plus affaire? On n'est plus "dans" le rite, mais dans un jeu ritualisé du rite; comme si nous étions gardès à distance de la scène des funérailles, comme si nous ne pouvions plus "directement" nous y investir. Comme si ces gestes qui supposent des croyances, qui engagent des gestes, ne pouvaient plus s'entendre qu'au second degré." (p. 38). Dans ce contexte, " comment croire à ce que l'on sait devoir faire croire? Comment participer de ce qu'on sait être une mise en scène?» (p. 77).

Or, insistera P. Baudry, l'imaginaire social est fondamental dans l'attribution d'une destinée post mortem de celui qui n'est plus, non pas au sens d'une simple destination religieuse, mais dans la construction cruciale d'une limite entre l'inhumain et I'humain. La faillite de l'imaginaire social rend difficilement perceptible cette limite et nuit au congédiement du mort. Cette confusion entre vivants et morts se manifeste à travers les progrès de la thanatopraxie qui viennent à la rescousse de notre incapacité à situer l'invisible. Au moment où le visage inhumain du défunt devrait nous aider à nous en séparer, le thanatopracteur "veut rapprocher le mort de ceux qui lui survivent, et permettre de le montrer "comme" de son vivant". ( $p$. 128). Selon le sociologue, "quand on croit que le mort s'est seulement absenté, qu'il va revenir tout à l'heure [...], on est dans la folie, dans l'irrespect de la séparation des morts et des vivants." (p. 129). Voilà une situation dangereuse qui, nous mentionne P. Baudry, affaiblit la capacité d'une société à ériger la limite fondatrice de l'espace humain

Le détour technique qui vise la production d'une image pacifiée de la mort en situant le défunt du côté des vivants, court-circuite non seulement la manœuvre culturelle d'une différenciation fondamentale entre vivants et morts, mais aussi notre capacité à entrer dans le deuil. Comment pourrait-on réussir à nous séparer de ce "vivant-mort" qui dort? Aux yeux de l'auteur, c'est cette confusion entre vivants et morts, entre l'inhumain et l'humain, qui fait défaut dans la ritualité funéraire contemporaine. Dans ces circonstances, et c'est ici que se situe l'enjeu fondamental de l'ouvrage, "comment une culture fondamentale parvient-elle à négocier autrement cet écart nécessaire, cette construction de l'ailleurs, et son articulation à ce monde?" ( $p$. 144). Une fois la question posée, le détour théorique achevé, le sociologue amène le lecteur au seuil de cette culture quotidienne où la mort ne saurait se taire.

À l'abri des regards, loin des funérariums et des cimetières, le souvenir du défunt échappe à toute forme de classification rationnelle, à la cristallisation de cette "dernière image" produite par le savoir technique. Dans la banalité des jours qui se suivent, une mélodie empreinte des moments forts du passé ou encore la vue d'un paysage ramène brusquement à la vie celui qui n'est plus. De même, I'histoire du mort continue de se raconter à travers la manipulation d'objets hautement symboliques. L'objet ressuscite la présence du défunt, compense la perte, mais bien plus, il établit la distance; il situe l'invisible dans notre monde. "C'est à cette condition dont nous savons depuis la vie ordinaire mettre en scène la représentation, que nous sommes protégés de la folie, que nous sommes protégés de la confusion." (p. 159). Discrètement, ces scènes de la vie quotidienne, qui se situent en marge des discours officiels, sont les manifestations tangibles de la manœuvre d'une culture fondamentale. 
L'apport de La place des morts à la littérature scientifique est considérable, non seulement pour la contemporanéité des questions de fond qui sont abordées, mais aussi à l'égard de la posture de l'auteur qui se situe en marge de l'esprit du temps. Au moment où plusieurs s'entendent à dire qu'il suffit "de parler de la mort" pour mieux l'intégrer, le sociologue Patrick Baudry questionne et critique brillamment les postulats sur lesquels se fonde cette tendance toute contemporaine à "l'humanisation" de la mort au détriment de la "socialisation". Nous fermons le livre en espérant trouver le temps d'y revenir..

\section{Sébastien St-Onge}

\section{Roger Régnier et Line Saint-Pierre Quand le deuil survient}

Montréal, Sciences et culture, 2000, 185 pages

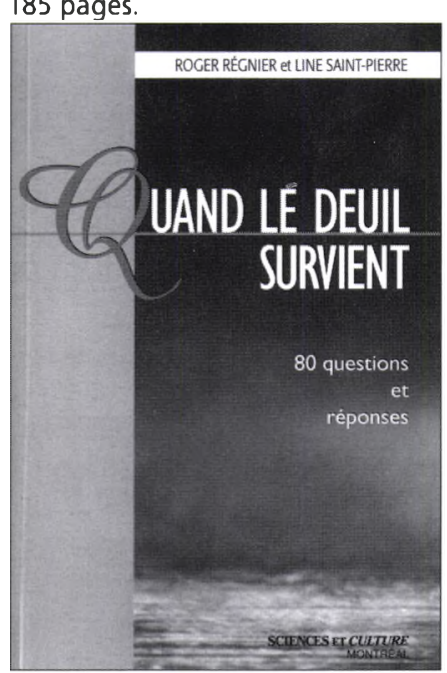

Les auteurs s'intéressent au domaine de la mort, de la perte et du deuil et ont fondé, en 1994, Deuil-Ressources, un organisme privé offrant des services d'accompagnement aux endeuillés, de formation aux intervenants et d'information auprès du grand public. Ces auteurs ont rassemblé dans ce livre les questions qui leur sont le plus souvent posées par les endeuillés. Comme mentionné dans l'avantpropos: "Un deuil soulève une multitude d'interrogations et d'inquiètudes qui rendent encore plus lourds la peine et les troubles causés par la perte". Le but visé est de procurer aux personnes en deuil l'information, le soutien et le réconfort dont elles ont tant besoin. Toutefois, reconnaissant que le deuil est un phénomène complexe, ils ne prétendent pas répondre à toutes les questions mais se sont délibérément limités à ce qu'ils considèrent comme étant l'essentiel.

Régnier et Saint-Pierre ont choisi de nous présenter le deuil sous la forme de 80 questionsréponses. Chacune des questions est traitée sur deux pages, ce qui exigeait de leur part des réponses claires, franches et concises. Dans le cas de questions très personnelles ou sujettes à différentes interprétations, ils proposent des pistes de réflexion afin d'aider le lecteur à formuler sa propre réponse. Le sommaire présente six sections: I Le phénomène du deuil; II - Les principales réactions provoquées par le deuil; III - Le déroulement du deuil; IV - Divers questionnements sur le deuil et sur ses répercussions; V - Le deuil et ses enfants; et VI L'aide aux endeuillés. Le tout suivi d'un glossaire donnant une définition simple des mots peu usuels.

Les trois premières sections, composées des questions 1 à 37, traitent principalement du processus de deuil et font un excellent survol des réactions émotives possibles, des attitudes contradictoires pouvant se manifester, des traitements aux antidépresseurs, etc. L'habileté démontrée par les auteurs pour présenter les points de vue divergents est fort impressionnante. Leur façon simple et directe d'aborder ces questions est à l'image de leur grande expérience, tant en recherche qu'en pratique, dans ce domaine.

La section IV, comprenant les questions 38 à 56 , est remarquable de par le large éventail des aspects abordés et des réponses succinctes et, surtout, des pistes de réflexions se rapportant à ces questions. Pour encourager les endeuillés, beaucoup d'informations fort utiles sont présentées telles que des renseignements sur comment faire face à la perte, le rôle des funérailles, les aspects pratiques des changements imposés au quotidien, etc. La section $V$, questions 57 à 69, traite plus spécifiquement des aspects touchant les jeunes enfants face à leur perte et, pour terminer, la section $\mathrm{VI}$, questions 70 à 80 , aborde l'aide aux endeuillés.

Malgré le fait que cette forme de présentation soit très utile et présente une belle créativité sur le deuil, deux points doivent être soulignés concernant le langage. Premièrement, le terme "successi " à la question 3, qu'entend-on par phases ou étapes du deuil? Les premiers mots de leur réponse sont:
"Ce sont les stades successifs du processus du deuil». II est évident ici que les quatre périodes identifiées par Régnier et Saint-Pierre existent mais non d'une façon si démarquée ou d'une aussi grande intensité. Le traitement de cette notion, issue des travaux sur le deuil de Kübler-Ross', est faussé par l'interprétation que les stages sont des entités distinctes et viables. On s'attend à ce que les endeuillés réagissent d'une façon définie à chacune des étapes qui ne se présentent pas toujours de manière cohérente. Ceci entraîne souvent chez les gens un comportement inapproprié car ils croient savoir où une personne en deuil en est rendue dans son processus et donc comment elle devrait maintenant réagir face à sa perte. Malgré les nombreuses critiques face à cette interprétation de la théorie des étapes du deuil, il n'en demeure pas moins que cette théorie est très invoquée par ceux qui souhaitent que l'endeuillé agisse en conformité avec le schéma plutôt que d'aborder le schéma comme une approche pour comprendre le processus de deuil d'un individu. Pour faire suite aux mises en garde de Osteirweis et al. ${ }^{2}$ concernant les dangers d'une idée préconçue des stages, Sanders ${ }^{3}$ et d'autres la remplacent par l'idée de phases, impliquant une certaine fluidité dans le processus puisque les symptômes d'une phase peuvent chevaucher la suivante ou même indiquer une régression à une phase précédente. Le fait d'intervertir ces deux termes ne donne rien si l'idée de progression n'est pas revue.

À la fin du texte, les auteurs reconnaissent cette fluidité dans le processus mais la façon catégorique dont elle est traitée au début démontre, je crois, qu'ils croient en une succession distincte de stages

Le deuxième point sous réserve touche le concept et l'utilisation du mot "résolution». La définition du glossaire est: au sens utilisé dans le texte, action de résoudre (résorber, faire disparaitre, trouver une solution) et son résultat. Malgré que le terme résolution soit très largement utilisé dans la littérature, je crois qu'il est nécessaire que l'on en cesse l'emploi dans le sens de solutionner un problème plutôt que celui d'aborder une période difficile de notre vie que l'on doit traverser graduellement pour mieux intégrer la perte. Si le deuil est perçu comme étant un problème à résoudre, la tension créée par la réalisation graduelle que ceci est impossible peut devenir un véri- table fardeau pour certains endeuillés.

Bref, en tenant compte de ces deux points, je crois que cet ouvrage contribue grandement à notre connaissance du deuil. Régnier et Saint-Pierre, faisant montre d'une belle créativité en proposant ces questions-réponses dans un langage clair et simple soutenu par un large éventail de données, nous proposent une excellente référence qui sera utile à "ceux et celles qui ont perdu un être cher, mais aussi à toute personne préoccupée par le deuil».

\section{Margaret C. Kiely \\ E. KÜBLER-ROSS, On Death and Dying New York, Macmillan, 1969 $M$. OSTEIRWEIS, E. SOLOMON, et $M$ GREEN (dir. publ.), Bereavement: Reactions, Consequences and Care Report of the Committee for the Study of Health, Consequences of the Stress of Bereavement, Institute of Medicine. National Academy of Sciences) Washington, DC, National Academy Press, 1964. \\ C. SANDERS, Grief: The Mourning After: Dealing with Adult Bereavement, New York, John Wiley, 1989.}

\section{BIBLIOGRAPHIE INDICATIVE}

Caroline Munger

\section{CULTURES CONTEMPORAINES}

\section{Sociétés occidentales}

ALLIOT, M. M., La grande peur des classes moyennes, Paris, Table ronde, 1996, 225

ARNAUD, G., Le salaire de la peur, Paris, Julliard, 1950, 194 pages.

BELLAVANCE, É., (dir. publ.), Éditions MNH, Beauport, 2000, 160 pages.

BEHR, E., Une Amérique qui fait peur, Paris, Plon, 1995, 324 pages. HALLAM, R.S., "Some Constructionist Observations on Anxiety and his History", dans T.R. SARBIN et J.I. KITSUSE (dir. publ.), Constructing the Social, London, Sage Publications Ltd., 1994.

MONGIN, O., La peur du vide: essai sur les passions démocratiques, Paris, Éd. du Seuil, 1991, 276 pages. ROJZMAN, C., La peur, la haine et la démocratie: introduction à une thérapie sociale, Paris, Desclée de Brouwer, 1992, 151 pages. 